\title{
SPECTRUM DETOUR GRAF n-PARTISI KOMPLIT
}

\author{
Desy Norma Puspita Dewi \\ Jurusan Matematika UIN Maulana Malik Ibrahim Malang \\ e-mail:phyta_23@yahoo.co.id
}

\begin{abstract}
ABSTRAK
Matriks detour dari graf $G$ adalah matriks yang elemen ke-(i,j) merupakan panjang lintasan terpanjang antara titik $v_{i}$ ke titik $v_{j}$ di $G$. Himpunan nilai eigen matriks detour dari graf terhubung langsung $G$ adalah spectrum detour. Spectrum detour dari graf $G$ biasanya dinotasikan dengan $\operatorname{spec}_{D D}(G)$. Dalam artikel ini, hanya menentukan spectrum detour graf $n$-partisi komplit $\left(K_{n, n+1, n+2, \ldots, n+m}\right)$, dan graf 3partisi komplit $\left(K_{2,2, n}\right)$. Dalam menentukan spectrum detour graf tersebut dengan cara menggambar pola grafnya, mencari matriks detournya, setelah itu dicari nilai eigen dan vektor eigen dari matriks tersebut, sehingga diperoleh pola (konjektur) spectrum detour, kemudian merumuskan konjektur sebagai teorema yang dilengkapi dengan bukti-bukti.
\end{abstract}

Kata kunci: Grafn-Partisi Komplit, Matriks Detour, dan Spectrum,

ABSTRACT

The detour matrices of a graph is for its $(i, j)$ entry the length of the longest path between vertices $v_{i}$ to $v_{j}$ of $G$. Set of detour matrices eigenvalues of a connected graph $G$ are detour spectrum. Detour spectrum of $G$, denoted by $\operatorname{spec}_{D D}(G)$. In this article, only determination of detour spectrum of complete $n$ partition graph $\left(K_{n, n+1, n+2, \ldots, n+m}\right)$, and complete 3-partition graph $\left(K_{2,2, n}\right)$. Determination of spectrum detour graph are picturing model graph, then finding detour matrices, after that finded eigenvalues and eigenvectors from that matrices, with the result that detour spectrum obtained model of detour spectrum, end then formulate the model as theorem with its prove.

Keywords: Complete n- Partitions Graph, Detour Matrices, and Spectrum

\section{PENDAHULUAN}

Graf $G$ adalah pasangan $(V(G), E(G))$ dengan $V(G)$ adalah himpunan tidak kosong dan berhingga dari objek-objek yang disebut titik (vertex), dan $E(G)$ adalah himpunan (mungkin kosong) pasangan tak berurutan dari titik-titik berbeda di $V(G)$ yang disebut sisi (edge).

Misalkan terdapat suatu graf $G$, dari suatu graf tersebut dibentuk matriks adjacency atau matriks keterhubungan. Matriks adjacency merupakan matriks simetri. Matriks adjacency dapat dirubah menjadi matriks detour, yang unsur-unsur ke-- $(i, j)$ merupakan panjang lintasan terpanjang antara titik $i$ dan $j$. Setelah dibentuk menjadi matriks detour, maka dapat dicari nilai eigen dan vektor eigen dari matriks tersebut.

Biasanya spectrum graf dibentuk dari nilai eigen dari matriks terhubung langsung. Dalam pengertian, nilai eigen dari graf $G$ dinotasikan dengan $\lambda_{i}, \quad i=1,2, \ldots, n$ dan spectrum ditulis dengan $\operatorname{spec}(G)$. Matriks detour didefinisikan $\mathrm{DD}=\mathrm{DD}(G)$ dari $\mathrm{G}$ sehingga unsur ke $(i, j)$ adalah panjang lintasan terpanjang antara titik i dan j. Nilai eigen dari $\mathrm{DD}(G)$ disebut DD-nilai eigen dari
G dan membentuk DD-spectrum dari G, dinotasikan dengan $\operatorname{spec}_{\mathrm{DD}}(\mathrm{G})$. Karena matriks detour simetris, semua nilai eigen $\mu_{\mathrm{i}}, i=1,2, \ldots, n$ adalah real dan dapat diberi label $\mu_{1} \geq \mu_{2} \geq \cdots \geq$ $\mu_{\mathrm{n}}$. Jika $\mu_{\mathrm{i}_{1}} \geq \mu_{\mathrm{i}_{2}} \geq \cdots \geq \mu_{\mathrm{i}_{\mathrm{g}}}$ adalah nilai eigen dari matriks detour, maka DD-spectrum dapat ditulis sebagai

$$
\operatorname{spec}_{D D}(G)=\left[\begin{array}{llll}
\mu_{\mathrm{i}_{1}} & \mu_{\mathrm{i}_{2}} & \ldots & \mu_{\mathrm{i}_{\mathrm{g}}} \\
m_{1} & m_{2} & \ldots & m_{g}
\end{array}\right]
$$

di mana $m_{j}$ menyatakan banyaknya basis untuk ruang vektor eigen $\mathrm{m}_{\mathrm{i}_{\mathrm{j}}}$ dan $\mathrm{m}_{1}+\mathrm{m}_{2}+\cdots+\mathrm{m}_{j}=$ n. (Ayyaswamy dan Balachandran, 2010:250).

\section{KAJIAN TEORI}

\section{Graf}

\section{Definisi 1}

Graf $G$ adalah pasangan himpunan $(V, E)$ dengan $V$ adalah himpunan tidak kosong dan berhingga dari obyek-obyek yang disebut sebagai titik dan $E$ adalah himpunan (mungkin kosong) pasangan tak berurutan dari titik-titik berbeda di $G$ yang disebut sebagai sisi (Chartrand dan Lesniak, 1986:4). 
Sehingga jika $G=(V(G), E(G))$, maka $V(G)=\left\{v_{1}, v_{2}, \ldots, v_{n}\right\}$ dan $E(G)=\left\{e_{1}, e_{2}, \ldots\right.$, $\left.e_{n}\right\}$, dimana $v_{i} \in V(G), i=1,2, \ldots, n$ disebut titik (vertex) dan $e_{j} \in E(G), j=1,2, \ldots, m$ disebut sisi (edge).

\section{Adjacent dan Incident}

Sisi $e=u v$ dikatakan menghubungkan titik $u$ dan $v$. Jika $e=u v$ adalah sisi $\operatorname{di} \operatorname{graf} G$, maka $u$ dan $v$ disebut terhubung langsung (adjacent). $v$ dan $e$ serta $u$ dan $e$ disebut terkait langsung (incident). Titik $u$ dan $v$ disebut ujung dari $e$. Dua sisi berbeda $e_{1}$ dan $e_{2}$ disebut terhubung langsung jika terkait langsung pada titik yang sama. Untuk selanjutnya sisi $e=(u, v)$ ditulis $e=u v$ (Abdussakir, dkk, 2009:6).

\section{Graf Komplit}

\section{Definisi 2}

Graf komplit (complete graph) adalah graf dengan dua titik yang berbeda saling terhubung langsung (adjacent). Graf komplit dengan $n$ titik dinyatakan dengan $K_{n}$ (Chartrand dan Lesniak, 1986:9).

\section{Definisi 3}

Graf $G$ dikatakan partisi $n$-komplit jika $G$ adalah graf partisi-n dengan himpunan partisi $V_{1}, V_{2}, \ldots, V_{n}$ sehingga jika $u \neq V_{i}$ dan $v \neq V_{j}, i \neq j$, maka $u v \neq E(G)$. Maka graf ini dinotasikan dengan $K_{p_{1}, p_{2}, \ldots, p_{n}}$ (Abdussakir, dkk. 2009:23).

\section{Graf Terhubung \\ Definisi 4}

Misalkan $G$ graf. Misalkan $u$ dan $v$ adalah titik pada $G$. Jalan (trail) $u v$ pada $G$ yang dinotasikan $W$ adalah barisan berhingga yang berganti $W: u=v_{0}, e_{1}, v_{1}, e_{2}, v_{2}, \ldots$, $e_{n}, v_{n}=v$ antara titik dan sisi yang diawali dan diakhiri dengan titik dengan $e_{i}=v_{i-1} v_{i}$ adalah sisi di $G$ untuk $i=1,2, \ldots, n$. $v_{0}$ disebut titik awal dan $v_{n}$ disebut titik akhir. Titik $v_{0}, v_{1}, v_{2}, \ldots, v_{n}$ disebut titik internal, dan $n$ menyatakan panjang dari $W$. Jika $v_{0} \neq v_{n}$, maka $W$ disebut jalan terbuka. Jika $v_{0}=v_{n}$ maka $W$ disebut jalan tertutup. Jalan yang tidak mempunyai sisi disebut jalan trivial (Abdussakir, dkk. 2009:49).

\section{Nilai Eigen dan Vector Eigen}

\section{Definisi 5}

Misalkan $A$ sebuah matrik $n \times n$. Bilangan $\lambda$ disebut nilai eigen (eigenvalue) dari $A$ jika terdapat vektor tidak nol $v \in F^{n}$ sedemikian sehingga $A x=\lambda \mathrm{x}$. Kemudian vektor $x$ disebut vektor eigen (eigenvector) dari $A$ yang berpasangan ke nilai eigen $\lambda$ (Jain \& Gunawardena, 2004:151).

\section{Spectrum Graf}

Misalkan terdapat suatu graf $G$, dari suatu graf tersebut dibentuk matriks adjacency atau matriks keterhubungan. Matriks adjacency merupakan matriks simetri. Matriks adjacency dapat dirubah menjadi matriks detour, yang unsur-unsur ke- $(i, j)$ merupakan panjang lintasan terpanjang antara titik $i$ dan $j$. Setelah dibentuk menjadi matriks detour, maka dapat dicari nilai eigen dan vektor eigen dari matriks tersebut.

Misalkan $G$ graf berorder $p$ dan $A$ matriks keterhubungan dari graf $G$. Suatu vektor tak nol $x$ disebut vektor eigen (eigen vector) dari $A$ jika $A x$ adalah suatu kelipatan skalar dari $x$, yakni $A x=\lambda x$, untuk sebarang skalar $\lambda$. Skalar $\lambda$ disebut nilai eigen (eigen value) dari $A$, dan $x$ disebut sebagai vektor eigen dari $A$ yang bersesuaian dengan $\lambda$. Untuk menentukan nilai eigen dari matriks $A$, persamaan $(A x=\lambda I x) x$ ditulis kembali dalam bentuk

$(A-\lambda I) x=0$,
dengan $I \quad$ matriks identitas berordo p. Persamaan ini akan mempunyai solusi tak nol jika dan hanya jika

$$
\operatorname{det}(A-\lambda I) x=0
$$

Persamaan $\operatorname{det}(A-\lambda I)=0$ akan menghasilkan persamaan polinomial dalam variabel $\lambda$ dan disebut persamaan karakteristik dari matriks $A$. Skalar-skalar $\lambda$ yang memenuhi persamaan karakteristik ini tidak lain adalah nilai-nilai eigen dari matriks $A$.

Misalkan $\lambda_{1}, \lambda_{2}, \ldots, \lambda_{n}$ adalah nilai eigen berbeda dari $A$, dengan $\lambda_{1}, \lambda_{2}, \ldots, \lambda_{n}$, dan misalkan $m \lambda_{1}, m \lambda_{2}, \ldots, m \lambda_{n}$ adalah banyaknya basis untuk ruang vektor eigen masingmasing $l_{i}$, maka matriks berordo $(2 \times n)$ yang memuat $\lambda_{1}, \lambda_{2}, \ldots, \lambda_{n}$ pada baris pertama dan $m \lambda_{1}, m \lambda_{2}, \ldots, m \lambda_{n}$ pada baris kedua disebut spectrum graf $G$, dan dinotasikan dengan $\operatorname{spec}(G)$. Jadi spectrum graf $G$ dapat ditulis dengan

$$
\operatorname{spec}(G)=\left[\begin{array}{cccc}
\lambda_{1} & \lambda_{2} & \cdots & \lambda_{n} \\
m \lambda_{1} & m \lambda_{2} & \cdots & m \lambda_{n}
\end{array}\right]
$$

(Abdussakir, dkk, 2009:82-83).

\section{Graf dalam Matriks Detour}

Matriks detour didefinisikan $D D=$ $D D(G)$ dari $G$ sehingga unsur atau entry $(i, j)$ adalah panjang lintasan terpanjang antara titik $i$ dan $j$. Nilai eigen dari $D D(G)$ disebut $D D$ nilai eigen dari $G$ dan membentuk $D D$ spectrum dari $G$, yang dinotasikan dengan $\operatorname{spec}_{D D}(G)$. Selama matriks detour simetris, semua nilai eigen $\mu_{i}, i=1,2, \ldots, n$ adalah real dan dapat diberi label $\mu_{1} \geq \mu_{2} \geq \cdots \geq \mu_{n}$. Jika $\mu_{i_{1}} \geq \mu_{i_{2}} \geq \cdots \geq \mu_{i_{g}}$ adalah nilai eigen dari matriks detour, maka $D D$-spectrum dapat ditulis sebagai 


$$
\operatorname{spec}_{D D}(G)=\left[\begin{array}{llll}
\mu_{\mathrm{i}_{1}} & \mu_{\mathrm{i}_{2}} & \cdots & \mu_{\mathrm{i}_{\mathrm{g}}} \\
m_{1} & m_{2} & \cdots & m_{g}
\end{array}\right]
$$

di mana $m_{j}$ menunjukkan banyaknya basis untuk ruang vektor eigen dalam $\mu_{i_{j}}$ dan tentunya $m_{1}+m_{2}+\ldots+m_{g}=n$.

(Ayyaswamy dan Balachandran, 2010)

\section{PEMBAHASAN}

\section{Spectrum Detour dari Graf $n$-Partisi} Komplit $\left(K_{n, n+1, n+2, \ldots, n+m}\right)$

Pembahasan spectrum detour dari graf $n$ - partisi komplit $\left(K_{n, n+1, n+2, \ldots, n+m}\right)$, dibatasi pada $n \geq 2, m \geq 1$ dan $n, m \in N$.

\subsection{Spectrum Detour Graf 3-Partisi Komplit} $K_{2,3,4}$

$$
\operatorname{spec}_{D D}\left(K_{2,3,4}\right)=\left[\begin{array}{cc}
64 & -8 \\
1 & 8
\end{array}\right]
$$

\subsection{Spectrum Detour Graf 4-Partisi Komplit} $K_{2,3,4,5}$

$$
\operatorname{spec}_{D D}\left(K_{2,3,4,5}\right)=\left[\begin{array}{cc}
169 & -13 \\
1 & 13
\end{array}\right]
$$

1.3 Spectrum Detour Graf 5-Partisi Komplit $K_{2,3,4,5,6}$

$$
\operatorname{spec}_{D D}\left(K_{2,3,4,5}\right)=\left[\begin{array}{cc}
361 & -19 \\
1 & 19
\end{array}\right]
$$

\subsection{Spectrum Detour Graf 6-Partisi Komplit}

$K_{2,3,4,5,6,7}$

$$
\operatorname{spec}_{D D}\left(K_{2,3,4,5}\right)=\left[\begin{array}{cc}
676 & -26 \\
1 & 26
\end{array}\right]
$$

\section{Teorema 1:}

Jika $\left(K_{n, n+1, n+2, \ldots n+m}\right)$ adalah graf $n$-partisi komplit dengan $n \geq 2, m \geq 1 ; n, m \in N$ dan $p=n+(n+1)+(n+2)+\cdots+(n+m)$, maka:

$$
\begin{aligned}
\operatorname{spec}_{D D}\left(K_{n, n+1, n+2, \ldots n+m}\right) & \\
= & {\left[\begin{array}{cc}
(p-1)^{2} & -(p-1) \\
1 & (p-1)
\end{array}\right] }
\end{aligned}
$$

dimana $\operatorname{spec}_{D D}\left(K_{n, n+1, n+2, \ldots n+m}\right)$ adalah spectrum detour dari graf $n$-partisi komplit dan $n$ bilangan asli.

Bukti:

Misalkan $D D\left(K_{n, n+1, n+2, \ldots n+m}\right)$ adalah matrik detour adjacent dari $\left(K_{n, n+1, n+2, \ldots n+m}\right)$, maka

$$
\begin{aligned}
& D D\left(K_{n, n+1, n+2, \ldots n+m}\right) \\
& =\left[\begin{array}{ccccc}
0 & p-1 & p-1 & \ldots & p-1 \\
p-1 & 0 & p-1 & \ldots & p-1 \\
p-1 & p-1 & 0 & \ldots & p-1 \\
\vdots & \vdots & \vdots & \ddots & \vdots \\
p-1 & p-1 & p-1 & \ldots & 0
\end{array}\right]
\end{aligned}
$$

Dari matriks detour adjacent di atas, maka akan dicari nilai eigennya dengan menentukan $\operatorname{det}\left(\lambda I-D D\left(K_{n, n+1, n+2, \ldots n+m}\right)\right)=0$

$$
\begin{gathered}
\mid\left[\begin{array}{ccccc}
1 & 0 & 0 & \ldots & 0 \\
0 & 1 & 0 & \ldots & 0 \\
0 & 0 & 1 & \ldots & 0 \\
\vdots & \vdots & \vdots & \ddots & \vdots \\
0 & 0 & 0 & \ldots & 1
\end{array}\right]-\left[\begin{array}{ccccc}
0 & p-1 & p-1 & \ldots & p-1 \\
p-1 & 0 & p-1 & \ldots & p-1 \\
p-1 & p-1 & 0 & \ldots & p-1 \\
\vdots & \vdots & \vdots & \ddots & \vdots \\
p-1 & p-1 & p-1 & \ldots & 0
\end{array}||=0\right. \\
\left|\begin{array}{ccccc}
\lambda & -(p-1) & -(p-1) & \ldots & -(p-1) \\
-(p-1) & \lambda & -(p-1) & \ldots & -(p-1) \\
-(p-1) & -(p-1) & \lambda & \ldots & -(p-1) \\
\vdots & \vdots & \vdots & \ddots & \vdots \\
-(p-1) & -(p-1) & -(p-1) & \ldots & \lambda
\end{array}\right|=0
\end{gathered}
$$

Kita kalikan matriks di atas dengan $\frac{1}{-(p-1)}$, sehingga diperoleh

$$
\left|\begin{array}{ccccc}
\frac{\lambda}{-(p-1)} & 1 & 1 & \ldots & 1 \\
1 & \frac{\lambda}{-(p-1)} & 1 & \cdots & 1 \\
1 & 1 & \frac{\lambda}{-(p-1)} & \cdots & 1 \\
\vdots & \vdots & \vdots & \ddots & \vdots \\
1 & 1 & 1 & \cdots & \frac{\lambda}{-(p-1)}
\end{array}\right|=0
$$

Dimisalkan $\lambda^{\prime}=\left(\frac{\lambda}{p-1}\right)$, maka

$$
\left|\begin{array}{ccccc}
-\lambda^{\prime} & 1 & 1 & \ldots & 1 \\
1 & -\lambda^{\prime} & 1 & \ldots & 1 \\
1 & 1 & -\lambda^{\prime} & \ldots & 1 \\
\vdots & \vdots & \vdots & \ddots & \vdots \\
1 & 1 & 1 & \ldots & -\lambda^{\prime}
\end{array}\right|=0
$$

Melalui operasi basis elementer, matriks $\operatorname{det}\left(\lambda I-D D\left(K_{n, n+1, n+2, \ldots n+m}\right)\right)$ direduksi menjadi matriks segitiga atas, sehingga diperoleh

$$
\left|\begin{array}{ccccc}
-\lambda^{\prime} & 1 & 1 & \ldots & 1 \\
0 & \frac{-\left(\lambda^{\prime 2}-1\right)}{\lambda^{\prime}} & 1 & \ldots & 1 \\
0 & 0 & \frac{-\left(\lambda^{\prime 2}-1\right)\left(\lambda^{\prime}-2\right)}{\lambda^{\prime}-1} & \ldots & 1 \\
\vdots & \vdots & \vdots & \ddots & \vdots \\
0 & 0 & 1 & \ldots & \frac{-\left(\lambda^{\prime^{2}}-(\mathrm{p}-2)(\mathrm{p}-1)\left(\lambda^{\prime}-(\mathrm{p}-1)\right)^{2}\right)}{\lambda^{\prime}-(\mathrm{p}-2)(\mathrm{p}-1)}
\end{array}\right|=0
$$

Sehingga $\operatorname{det}\left(\lambda I-D D\left(K_{n, n+1, n+2, \ldots n+m}\right)\right)$ tidak lain adalah hasil perkalian diagonal matriks segitiga atas tersebut, sehingga diperoleh

$$
\begin{array}{r}
\operatorname{det}\left(\lambda I-D D\left(K_{n, n+1, n+2, \ldots n+m}\right)\right) \\
=\left(\lambda^{\prime}-(p-1)\right)\left(\lambda^{\prime}+1\right)^{p-1}
\end{array}
$$

Karena $\operatorname{det}\left(\lambda I-D D\left(K_{n, n+1, n+2, \ldots n+m}\right)\right)=0$, maka

$$
\left(\lambda^{\prime}-(\mathrm{p}-1)\right)\left(\lambda^{\prime}+1\right)^{\mathrm{p}-1}=0
$$

Sehingga didapat nilai eigen $\lambda^{\prime}=(p-1)$ atau $\lambda^{\prime}=-1$, karena $\lambda^{\prime}=\frac{\lambda}{-(\mathrm{p}-1)}$ maka nilai eigennya diperoleh $\lambda=(p-1)$ atau $\lambda=-1$

$$
\begin{array}{lc}
\frac{\lambda}{(\mathrm{p}-1)}=(\mathrm{p}-1) & \frac{\lambda}{(\mathrm{p}-1)}=-1 \\
\lambda=(\mathrm{p}-1)^{2} & \lambda=-(\mathrm{p}-1)
\end{array}
$$

Sedangkan untuk vektor eigennya, yaitu

$$
A x=\lambda x=0
$$




$$
\left[\begin{array}{ccccc}
-\lambda & 1 & 1 & \cdots & 1 \\
1 & -\lambda & 1 & \cdots & 1 \\
1 & 1 & -\lambda^{\prime} & \cdots & 1 \\
\vdots & \vdots & \vdots & \ddots & \vdots \\
1 & 1 & 1 & \cdots & -\lambda^{\prime}
\end{array}\right]\left[\begin{array}{c}
x_{1} \\
x_{2} \\
x_{(p-1)} \\
\vdots \\
x_{p}
\end{array}\right]=\left[\begin{array}{c}
0 \\
0 \\
0 \\
\vdots \\
0
\end{array}\right]
$$

Kemudian, akan dibuktikan bahwa untuk $\lambda=(n-1)^{2}$ akan didapatkan banyaknya basis vektor eigen adalah 1 .

Untuk $\lambda=-(\mathrm{p}-1)$ akan didapatkan

$$
\begin{aligned}
& {\left[\begin{array}{ccccc}
\frac{(p-1)^{2}}{-(p-1)} & 1 & 1 & \cdots & 1 \\
1 & \frac{(p-1)^{2}}{-(p-1)} & 1 & \cdots & 1 \\
1 & 1 & \frac{(p-1)^{2}}{-(p-1)} & \cdots & 1 \\
\vdots & \vdots & \vdots & \ddots & \vdots \\
1 & 1 & 1 & \cdots & \frac{(p-1)^{2}}{-(p-1)}
\end{array}\right]\left[\begin{array}{c}
x_{1} \\
x_{2} \\
x_{(p-1)} \\
\vdots \\
x_{p}
\end{array}\right]=\left[\begin{array}{c}
0 \\
0 \\
0 \\
\vdots \\
0
\end{array}\right]} \\
& {\left[\begin{array}{ccccc}
-(p-1) & 1 & 1 & \cdots & 1 \\
1 & -(p-1) & 1 & \cdots & 1 \\
1 & 1 & -(p-1) & \cdots & 1 \\
\vdots & \vdots & \vdots & \ddots & \vdots \\
1 & 1 & 1 & \cdots & -(p-1)
\end{array}\right]\left[\begin{array}{c}
x_{1} \\
x_{2} \\
x_{(p-1)} \\
\vdots \\
x_{p}
\end{array}\right]=\left[\begin{array}{c}
0 \\
0 \\
0 \\
\vdots \\
0
\end{array}\right]}
\end{aligned}
$$

Dengan mereduksi matriks di atas menjadi bentuk eselon tereduksi baris, aka didapatkan

$$
\left[\begin{array}{ccccc}
1 & 0 & 0 & \cdots & -1 \\
0 & 1 & 0 & \cdots & -1 \\
0 & 0 & 1 & \cdots & -1 \\
\vdots & \vdots & \vdots & \ddots & \vdots \\
0 & 0 & 0 & \cdots & 0
\end{array}\right]\left[\begin{array}{c}
x_{1} \\
x_{2} \\
x_{(p-1)} \\
\vdots \\
x_{p}
\end{array}\right]=\left[\begin{array}{c}
0 \\
0 \\
0 \\
\vdots \\
0
\end{array}\right]
$$

Kemudian didapat

$$
x_{1}=x_{p}, x_{2}=x_{p}, \ldots, x_{p-1}=x_{p}
$$

Sehingga diperoleh $x_{1}=x_{2}=\cdots=x_{p-1}=x_{p}$. Misal $x_{p}=s$ maka vektor eigennya adalah

$$
S_{1}=\left[\begin{array}{c}
x_{1} \\
x_{2} \\
\vdots \\
x_{(p-1)} \\
x_{p}
\end{array}\right]=\left[\begin{array}{c}
s \\
s \\
\vdots \\
s \\
s
\end{array}\right]=S\left[\begin{array}{c}
1 \\
1 \\
\vdots \\
1 \\
1
\end{array}\right]
$$

Jadi didapatkan banyaknya basis ruang eigen untuk $\lambda=(p-1)^{2}$ adalah 1 .

Untuk $\lambda=-(\mathrm{p}-1)$ akan didapatkan

$$
\begin{aligned}
& {\left[\begin{array}{ccccc}
\frac{-(p-1)}{-(p-1)} & 1 & 1 & \cdots & 1 \\
1 & \frac{-(p-1)}{-(p-1)} & 1 & \cdots & 1 \\
1 & 1 & \frac{-(p-1)}{-(p-1)} & \cdots & 1 \\
\vdots & \vdots & \vdots & \ddots & \vdots \\
1 & 1 & 1 & \cdots & \frac{-(p-1)}{-(p-1)}
\end{array}\right]\left[\begin{array}{c}
x_{1} \\
x_{2} \\
x_{(p-1)} \\
\vdots \\
x_{p}
\end{array}\right]=\left[\begin{array}{c}
0 \\
0 \\
0 \\
\vdots \\
0
\end{array}\right]} \\
& {\left[\begin{array}{ccccc}
1 & 1 & 1 & \cdots & 1 \\
1 & 1 & 1 & \cdots & 1 \\
1 & 1 & 1 & \cdots & 1 \\
\vdots & \vdots & \vdots & \ddots & \vdots \\
1 & 1 & 1 & \cdots & 1
\end{array}\right]\left[\begin{array}{c}
x_{1} \\
x_{2} \\
x_{(p-1)} \\
\vdots \\
x_{p}
\end{array}\right]=\left[\begin{array}{c}
0 \\
0 \\
0 \\
\vdots \\
0
\end{array}\right]}
\end{aligned}
$$

Dengan mereduksi matriks di atas menjadi bentuk eselon tereduksi baris, maka didapatkan

$$
\left[\begin{array}{ccccc}
1 & 1 & 1 & \cdots & 1 \\
0 & 0 & 0 & \cdots & 0 \\
0 & 0 & 0 & \cdots & 0 \\
\vdots & \vdots & \vdots & \ddots & \vdots \\
0 & 0 & 0 & \cdots & 0
\end{array}\right]\left[\begin{array}{c}
x_{1} \\
x_{2} \\
x_{(p-1)} \\
\vdots \\
x_{p}
\end{array}\right]=\left[\begin{array}{c}
0 \\
0 \\
0 \\
\vdots \\
0
\end{array}\right]
$$

Kemudian didapat $x_{1}+x_{2}+\cdots+x_{(p-1)}+x_{p}=0$ Sehingga diperoleh $x_{1}=-x_{2}-\cdots-x_{(p-1)}-x_{p}$. Maka vektor eigennya adalah

$$
S_{2}=\left[\begin{array}{c}
x_{1} \\
x_{2} \\
\vdots \\
x_{(p-1)} \\
x_{p}
\end{array}\right]=\left[\begin{array}{c}
x_{2}-\cdots-x_{(p-1)}-x_{p} \\
x_{p} \\
x_{(p-1)} \\
\vdots \\
x_{2}
\end{array}\right]
$$

Jadi didapatkan banyaknya basis ruang eigen untuk $\lambda=-(p-1)$ adalah $(p-1)$.

Jadi terbukti bahwa

$\operatorname{spec}_{D D}\left(K_{n, n+1, n+2, \ldots n+m}\right)=\left[\begin{array}{cc}(p-1)^{2} & -(p-1) \\ 1 & (p-1)\end{array}\right]$.

2. Spectrum Detour dari Graf 3-Partisi Komplit $K_{2,2, n}$

Pembahasan spectrum detour dari graf 3- partisi komplit $K_{2,2, n}$ dibatasi pada $n \geq 5$.

2.1 Spectrum Detour Graf 3-Partisi Komplit $K_{2,2,5}$

$$
\operatorname{spec}_{D D}\left(K_{2,2,5}\right)=\left[\begin{array}{cccc}
25+\sqrt{1029} & 25-\sqrt{1029} & -6 & -8 \\
1 & 1 & 3 & 4
\end{array}\right]
$$

2.2 Spectrum Detour Graf 3-Partisi Komplit $K_{2,2,6}$

$$
\operatorname{spec}_{D D}\left(K_{2,2,6}\right)=\left[\begin{array}{cccc}
29+\sqrt{129} 7 & 29-\sqrt{1297} & -6 & -8 \\
1 & 1 & 3 & 5
\end{array}\right]
$$

2.3 Spectrum Detour Graf 3-Partisi Komplit $K_{2,2,7}$

$$
\operatorname{spec}_{D D}\left(K_{2,2,7}\right)=\left[\begin{array}{cccc}
33+\sqrt{1597} & 33-\sqrt{1597} & -6 & -8 \\
1 & 1 & 3 & 6
\end{array}\right]
$$

\subsection{Spectrum Detour Graf 3-Partisi Komplit} $K_{2,2,8}$

$$
\operatorname{spec}_{D D}\left(K_{2,2,8}\right)=\left[\begin{array}{cccc}
37+\sqrt{1929} & 33-\sqrt{1929} & -6 & -8 \\
1 & 1 & 3 & 7
\end{array}\right]
$$

\subsection{Spectrum Detour Graf 3-Partisi Komplit}

$K_{2,2,9}$

$$
\operatorname{spec}_{D D}\left(K_{2,2,9}\right)=\left[\begin{array}{cccc}
41+\sqrt{2293} & 41-\sqrt{2293} & -6 & -8 \\
1 & 1 & 3 & 8
\end{array}\right]
$$

\subsection{Spectrum Detour Graf 3-Partisi Komplit} $K_{2,2,10}$ $\operatorname{spec}_{D D}\left(K_{2,2,10}\right)=\left[\begin{array}{cccc}45+\sqrt{2689} & 45-\sqrt{2689} & -6 & -8 \\ 1 & 1 & 3 & 9\end{array}\right]$ 
Berdasarkan hasil spectrum detour dari graf 3-partisi komplit $\left(K_{2,2, n}\right)$ di atas, dapat diperoleh dugaan sementara bahwa bentuk umum dari spectrum detour adalah:

$\operatorname{spe}_{D D}\left(K_{2, n}\right)=\left[\begin{array}{cccc}(2(2+n)+2 n+1)+\sqrt{16 n^{2}+220 n+793} & (2(2+n)+2 n+1)-\sqrt{16 n^{2}+220 n+793} & -6 & -8 \\ 1 & 1 & 3 & n-1\end{array}\right]$

Dengan $n \geq 5$ dan $n$ adalah bilangan asli.

\section{PENUTUP}

\section{Kesimpulan}

Berdasarkan pembahasan mengenai spectrum detour dari graf $n$-partisi komplit, diperoleh kesimpulan: (a). Untuk graf $n$-partisi komplit $\left(K_{n, n+1, n+2, \ldots, n+m}\right)$ dengan $n \geq 2, m \geq$ $1 ; n, m \in N$ dan $p=n+(n+1)+(n+2)+\cdots+$ $(n+m)$, maka

$\operatorname{spec}_{D D}\left(K_{n, n+1, n+2, \ldots, n+m}\right)=\left[\begin{array}{cc}(p-1)^{2} & -(p-1) \\ 1 & (p-1)\end{array}\right]$,

(b). Untuk graf 3-partisi komplit $\left(K_{2,2, n}\right)$ dengan $n \geq 2, n \in N$

$\operatorname{spec}_{D D}\left(K_{2,2, n}\right)=\left[\begin{array}{cccc}(2(2+n)+2 n+1)+\sqrt{16 n^{2}+220 n+793} & (2(2+n)+2 n+1)-\sqrt{16 n^{2}+220 n+793} & -6 & -8 \\ 1 & 1 & 3 & n-1\end{array}\right]$

\section{Saran}

Pada artikel ini, penulis hanya memfokuskan pada spectrum detour yang digambarkan oleh dua bentuk graf $n$-partisi komplit yaitu graf $n$-partisi komplit $\left(K_{n, n+1, n+2, \ldots, n+m}\right)$ dan graf 3-partisi komplit
$\left(K_{2,2, n}\right)$. Pada bentuk graf 3-partisi komplit $\left(K_{2,2, n}\right)$ masih merupakan konjektur, sehingga perlu diselidiki lebih lanjut. Karena masih banyaknya bentuk dari graf ini, maka untuk penulisan skripsi selanjutnya diteliti pada graf lain.

\section{DAFTAR PUSTAKA}

[1] Abdussakir, dkk. 2009. Teory Graf : Topik Dasar untuk Tugas Akhir/Skripsi. Malang: UIN-Malang Press.

[2] Chartrand, G and Lesniak, L. 1986. Graph and Digraph: Second Edition California: A Division Wadsworth

[3] Ayyaswamy, S.K. dan Balachandran, S. (2010). "On Detour Spectra of Some Graphs". World Academy of Science, Enggineering and Technology.

(www.waset.org/journals/waset/v67/v6788.pdf. diakses 2 Februari 2011).

[4] Jain, S. K. 2004. Linear Algebra; An Interactive Approach. Australia: Thomson Learning. 